\title{
Activating transcription factor-4 promotes mineralization in vascular smooth muscle cells
}

\author{
Masashi Masuda, ${ }^{1}$ Shinobu Miyazaki-Anzai, ${ }^{1}$ Audrey L. Keenan, ${ }^{1}$ Yuji Shiozaki, ${ }^{1}$ Kayo Okamura, ${ }^{1}$ \\ Wallace S. Chick, ${ }^{2}$ Kristina Williams, ${ }^{2}$ Xiaoyun Zhao, ${ }^{2}$ Shaikh Mizanoor Rahman, ${ }^{3}$ Yin Tintut, ${ }^{4}$ \\ Christopher M. Adams, ${ }^{5}$ and Makoto Miyazaki ${ }^{1}$ \\ 'Division of Renal Diseases and Hypertension, Department of Medicine, and 'Department of Cell and Developmental \\ Biology, University of Colorado Denver, Aurora, Colorado, USA. ${ }^{3}$ Department of Nutritional Sciences, Texas Tech University, \\ Lubbock, Texas, USA. " Division of Cardiology, Department of Medicine, University of California, Los Angeles, California, \\ USA. ${ }^{5}$ Department of Internal Medicine, University of lowa, lowa City, lowa, USA
}

Emerging evidence indicates that upregulation of the ER stress-induced pro-osteogenic transcription factor ATF4 plays an important role in vascular calcification, a common complication in patients with aging, diabetes, and chronic kidney disease (CKD). In this study, we demonstrated the pathophysiological role of ATF4 in vascular calcification using global Atf4 KO, smooth muscle cell-specific (SMC-specific) Atf4 KO, and transgenic (TC) mouse models. Reduced expression of ATF4 in global ATF4-haplodeficient and SMC-specific Atf4 KO mice reduced medial and atherosclerotic calcification under normal kidney and CKD conditions. In contrast, increased expression of ATF4 in SMC-specific Atf4 TC mice caused severe medial and atherosclerotic calcification. We further demonstrated that ATF4 transcriptionally upregulates the expression of type III sodium-dependent phosphate cotransporters (PIT1 and PiT2) by interacting with C/EBP及. These results demonstrate that the ER stress effector ATF4 plays a critical role in the pathogenesis of vascular calcification through increased phosphate uptake in vascular SMCs.

Authorship note: M. Masuda and S. Miyazaki-Anzai contributed equally to this work.

Conflict of interest: The authors have declared that no conflict of interest exists.

Submitted: May 18, 2016 Accepted: October 5, 2016 Published: November 3, 2016

Reference information: JCI Insight. 2016;1(18):e88646. doi:10.1172/jci.insight.88646.

\section{Introduction}

Recent evidence is emerging that ER stress contributes to the pathogenesis of vascular calcification (1-10). The ER is a major site for the regulation of calcium and lipid homeostasis (11). ER stress is an integrated signal transduction pathway involved in the localization and folding of secreted and transmembrane proteins. A number of cellular stress conditions, including lipid accumulation, lead to the accumulation of unfolded proteins in the ER lumen. The three ER resident sensors, PKR-like endoplasmic reticulum kinase (PERK), inositol-requiring enzyme 1 (IRE1), and activating transcription factor 6 (ATF6) implement the ER stress response (12). Among the three pathways of the ER stress response, our previous studies strongly implicate the PERK-initiated pathway in the development of vascular calcification (7-10). Activation of PERK leads to the phosphorylation of the $\alpha$-subunit of eukaryotic initiation factor 2 (eIF2 $\alpha$ ), which inhibits assembly of the $80 \mathrm{~S}$ ribosome and protein synthesis. However, ATF4 is not affected by the translational attenuation of eIF2 $\alpha$ phosphorylation due to the existence of several small upstream open reading frames in its 5'-untranslated regions. These upstream open reading frames, which prevent translation of ATF4 under normal conditions, are bypassed only when eIF2 $\alpha$ is phosphorylated, thereby permitting translation (13-15). ATF4 is a pivotal transcription factor that mediates not only ER stress, but also osteoblastic differentiation (16-19). In the late stage of osteoblastic differentiation, ATF4 directly induces osteocalcin, an osteoblast-specific marker, and osterix, another essential transcription factor, through their osteo-specific elements (OSE) $(17,19)$. ATF4 is also required for preserving mature osteoblast functions, including the synthesis of collagen, the most abundant extracellular protein found in bones and calcified vasculatures (19). PERK/eIF2 $\alpha /$ ATF4 pathway-mediated ER stress is involved in osteoblast differentiation induced by bone morphogenetic protein-2 and other factors $(8,20)$. In addition, we and other groups recently reported ectopic ATF4 expression in rodent models of vascular calcification $(1,2,5,7,8,10)$. However, the in vivo role of ATF4 in the regulation of vascular calcification and vascular osteogenesis has yet to be determined. 
Vascular calcification is an independent predictor for the mortality and morbidity of patients with chronic kidney disease (CKD) $(4,21)$. Vascular calcification is classified into two major types, atherosclerotic and medial, both of which are frequently and simultaneously observed in CKD patients. Vascular calcification is a highly regulated process that resembles skeletal bone formation. Many key transcriptional regulators involved in skeletal osteogenesis, such as Msx-2, osterix, and Runx-2, are expressed in both calcified medial arterial layers and atherosclerotic plaques $(22,23)$. In addition, many causative hormonal factors (TNF- $\alpha$, FGF23/Klotho, and phosphorus) and lipid-derived factors (saturated fats and oxidized lipids) have been identified in vascular calcification (7-10, 24-29). We recently reported that these factors induce ATF4 activation through the ER stress response, resulting in osteogenic differentiation and mineralization of vascular smooth muscle cells (VSMCs) in vitro $(8,9)$. However, whether in vivo ATF4 activation causes ectopic vascular osteogenesis and the molecular mechanism by which ATF4 induces mineralization of VSMCs have not been determined. In this context, we determined the in vivo role of ATF4 in VSMCs in both atherosclerotic and medial calcification and its mechanisms by using several murine models with global ATF4 deficiency, smooth muscle cell-specific (SMC-specific) ATF4 deficiency, and SMC-specific ATF4 overexpression.

\section{Results}

Global ATF4 haplodeficiency attenuates medial calcification. The in vivo role of ATF4 in the regulation of vascular calcification and vascular osteogenesis has yet to be determined. Medial calcification is characterized by calcific deposits in, along, or within one or more elastic lamellae of the aortic medial layer and is more frequently observed than atherosclerotic calcification in patients with CKD (4).

To investigate whether global ATF4 deficiency affects medial calcification, global ATF4-deficient mice were generated by backcrossing to DBA/2J mice, which are susceptible to aortic medial calcification (10, 30). Since ATF4 biallelic KO mice on DBA/2J background are embryonic lethal, we used ATF4-haplodeficient $\left(A t f 4^{+/-}\right)$mice. DBA/2J Atf $4^{+/-}$mice and WT $\left(A t f 4^{+/+}\right)$mouse littermates were subjected to 5/6 nephrectomy (nx) to induce CKD. Sham operation was used as normal kidney condition (NKD). Atf4 ${ }^{+/-}$ mice had approximately 53\% lower aortic ATF4 mRNA levels than Atf4 ${ }^{+/+}$mice (data not shown). 5/6 nx increased levels of serum creatinine and phosphorus, indicators of CKD (Supplemental Figure 1, A and B; supplemental material available online with this article; doi:10.1172/jci.insight.88646DS1). CKD also increased serum cholesterol, whereas levels of serum triglyceride, calcium, and glucose decreased (Supplemental Figure 1, C-F). Serum creatinine, phosphorus, cholesterol, triglyceride, calcium, and glucose levels were not different between $A t f 4^{+/+}$and $A t f 4^{+/-}$mice under both NKD and CKD conditions (Supplemental Figure 1, A-F).

In the aortic sinus, $A t f 4^{+/-}$mice had $90 \%$ and $96 \%$ fewer calcified lesions under NKD and CKD conditions, respectively (Figure 1, A and B, and Supplemental Figure 1G). ATF4 haploinsufficiency reduced aortic calcium content by $71 \%$ under CKD conditions (Figure 1C). VSMC apoptosis and aortic macrophage infiltrations are known to contribute to vascular calcification (2, 5, 26, 31-34). Apoptotic cell death, as shown by immunofluorescence analysis, TUNEL positivity, and CHOP expression, was significantly increased by CKD and reduced by ATF4 haploinsufficiency (Figure 1, D-F). Macrophage infiltrations into the aorta were not observed in any of the groups (data not shown). CKD increased the expression of another ER stress marker, KDEL, in the medial layer of the aorta. ATF4 haploinsufficiency did not alter levels of aortic KDEL protein, suggesting that ER chaperones, such as GRP78 and GRP94, are not major targets of ATF4 in VSMCs (Supplemental Figure 1H).

$S M C$-specific ATF4 deficiency attenuates medial and atherosclerotic calcification. We next investigated whether SMC-specific ATF4 deficiency affects medial calcification. Atf4 conditional floxed (Atf4 $\left.{ }^{L / L}\right)$ and $S M M H C$ $C r e^{E R(T 2)}$ mice were backcrossed 10 times to DBA/2J mice. DBA/2J Atf4 $4^{\mathrm{L} / \mathrm{L}}$ mice were crossed with DBA/2J $S M M H C-C r e^{E R(T 2)}$ mice to generate SMC-Atf4 conditional (SMMHC-Cre $\left.{ }^{E R(T 2)} ; A t f 4^{\mathrm{L} / \mathrm{L}}\right)$ mice. DBA/2J SMCAtf4 conditional mice were intraperitoneally injected with vehicle or tamoxifen to generate control mice and DBA/2J SMC-Atf4 KO mice, respectively. Tamoxifen injection diminished protein expression of ATF4 in the aortic medial layers of SMC-Atf4 KO mice (Figure 2A). These mice were subjected to either sham operation or $5 / 6 \mathrm{nx}$. CKD, as expected, increased levels of serum creatinine and phosphorus (Supplemental Figure 2, A and B), whereas SMC-specific ATF4 deficiency did not affect levels of serum creatinine, phosphorus, calcium, cholesterol, or triglyceride (Supplemental Figure 2, A-E).

$\mathrm{CKD}$ induced medial calcification but not atherosclerosis in the aortic sinuses of DBA/2J mice (Figure 
A

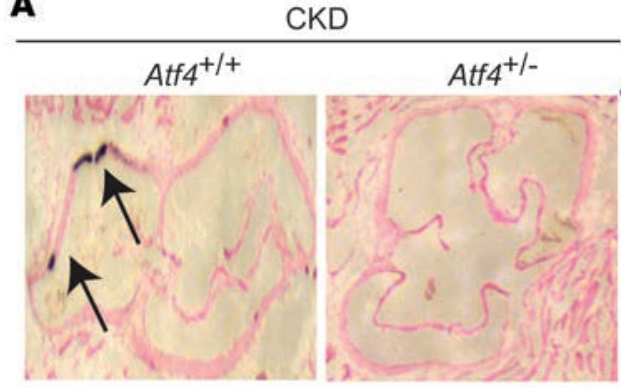

$\overline{100 \mu m}$
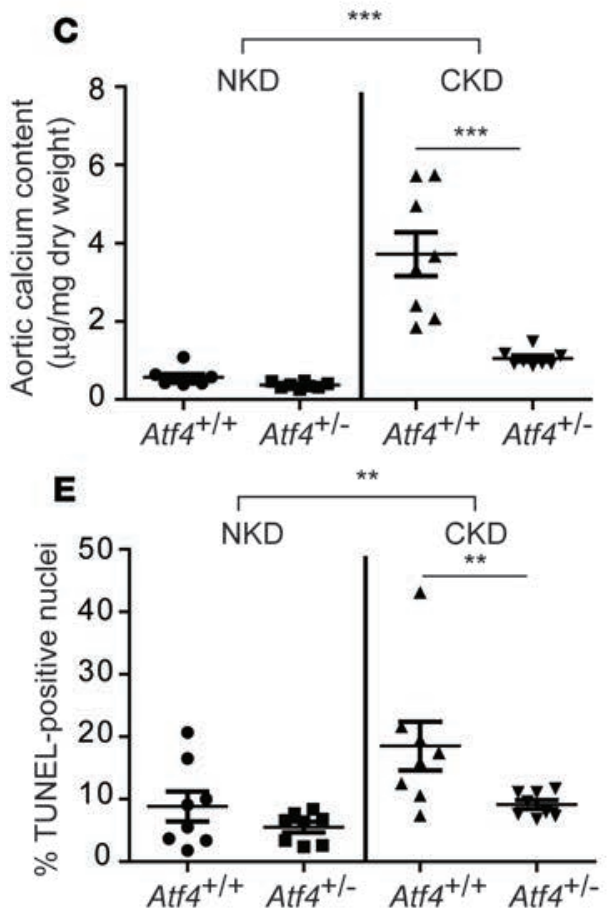

B

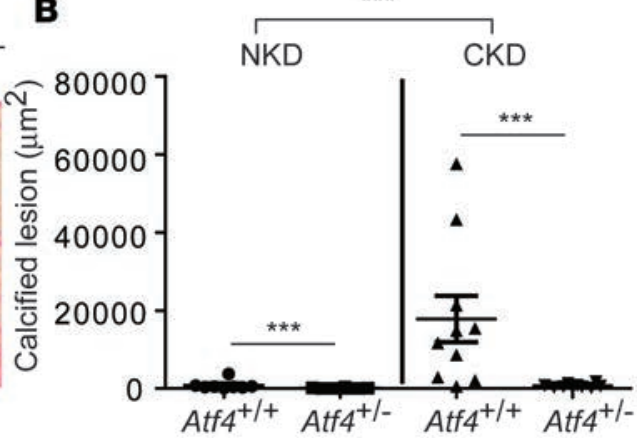

D

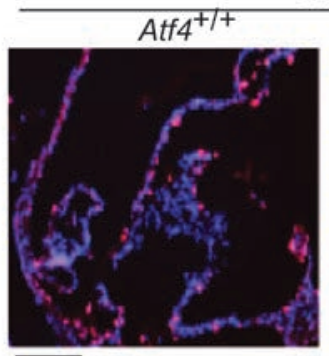

$\overline{100 \mu \mathrm{m}}$

$\mathbf{F}$

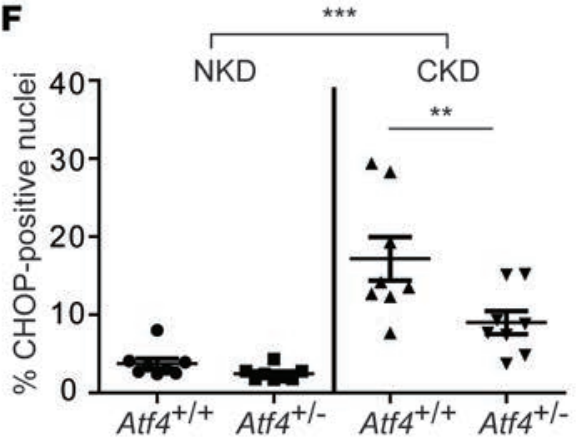

Figure 1. ATF4 haploinsufficiency attenuates medial calcification.

(A) Representative photographs (original magnification, $\times 10$ ) of the lesions in aortic sinuses stained with von Kossa from 8-week-old Atf $4^{+/+}$and Atf4 ${ }^{+/-}$mice $(n=8-10)$ on DBA/2 J background. Mice were subjected to either sham operation for normal kidney condition (NKD) or $5 / 6 n x$ for chronic kidney disease condition (CKD). The mice were sacrificed 12 weeks after the surgeries. (B) Quantitative analysis of calcified lesions in the aortic sinus. More NKD data are shown in Supplemental Figure 1G. (C) Aortic calcium content in Atf4+/- mice. (D) Representative micrographs show more TUNEL-positive signal (pink) in nuclei (blue) of aortic sinus lesions from $\mathrm{Atf4}^{+/+}$mice than from $\mathrm{Atf4}^{+/-}$ mice. (E) Quantitative analysis of TUNEL-positive nuclei (pink) conducted on lesions from Atf4 $4^{+/}$mice. (F) Immunofluorescence analysis of CHOP (pink) in the aortic sinuses of Atf4+/- mice. One-way ANOVA with a Student-Newman post-hoc test was used for comparison between Atf $4^{+/+}$ and $\mathrm{Atf4}^{+/-}$mice. Two-way ANOVA was used for comparison between NKD and CKD. ${ }^{* *} P<0.01,{ }^{* *} P<$ 0.001 . Scale bar: $100 \mu \mathrm{m}$.

2, B and C). SMC-Atf4 KO mice had significantly smaller calcified medial lesions than control mice under both NKD and CKD, by $81 \%$ and $88 \%$, respectively (Figure $2 \mathrm{C}$ and Supplemental Figure $2 \mathrm{~F}$ ). Aortic calcium content was also significantly lower in SMC-Atf4 KO mice under CKD (Figure 2D). mRNA levels of ER stress markers such as Atf4 and Chop and osteogenic makers such as alkaline phosphatase, osteopontin, and osteocalcin that were upregulated by CKD were attenuated in the medial layer of aortas of SMC-Atf4 KO mice (Supplemental Figure 2G). Consistent with global ATF4 haploinsufficiency, immunofluorescence analysis indicated that SMC-specific ATF4 deficiency reduced CKD-induced aortic apoptosis and CHOP and ATF4 expression (Figure 2, E and F, and Supplemental Figure 2, H and I).

To study the role of ATF4 in atherosclerotic calcification, we generated SMC-specific Atf4 KO mice on an ApoE-deficient background. Five-week-old SMMHC-CreER ${ }^{(T 2)} ; A t f 4^{L / L} ; A p o E^{-/-}$mice were intraperitoneally injected with vehicle or tamoxifen to generate control $A p o E^{-/-}$mice and SMC-specific Atf4 $\mathrm{KO} ; A p o E^{-1-}$ mice. Control and SMC-specific Atf4 KO; $A p o E^{-/-}$mice were subjected to either sham operation for NKD or $5 / 6 \mathrm{nx}$ for CKD at 8 weeks of age. After 12 weeks on a Western diet, control $A p o E^{-/}$ mice under NKD displayed mild atherosclerotic calcification, which was further worsened by CKD. However, SMC-specific ATF4 deficiency strongly inhibited vascular calcification under both NKD and CKD (Figure 3, A and B). Aortic calcium content was significantly lower in SMC-Atf4 KO mice than in control mice under CKD conditions (Figure 3C), supporting the histological analyses. Atherosclerotic formation (Figure 3D) and CD68-positive macrophage infiltrations into the aorta (data not shown) were not altered by SMC-specific ATF4 deficiency. There were no differences in body weight, serum cholesterol, triglyceride, phosphorus, calcium, glucose, and creatinine between control ApoE $E^{-1-}$ mice 
A

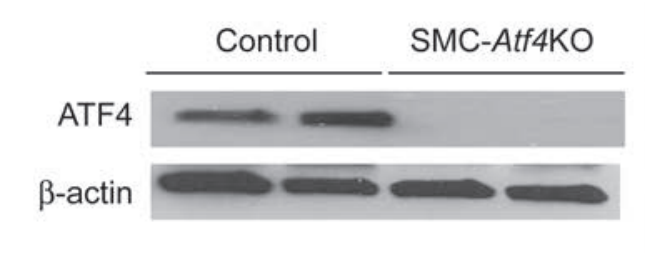

C

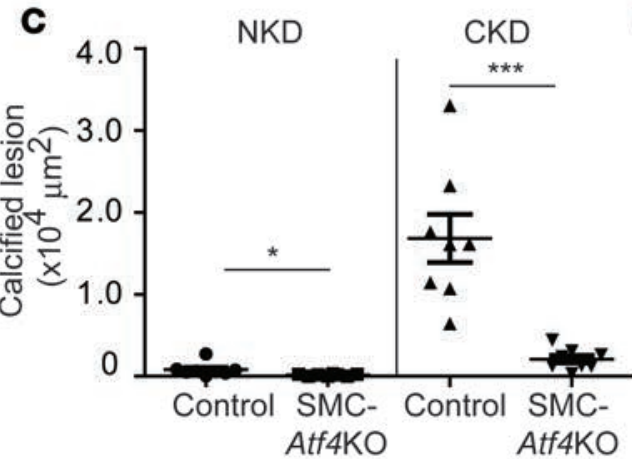

E

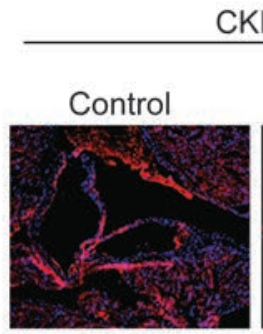

CKD

\begin{abstract}
SMC-
\end{abstract} Atf4KO
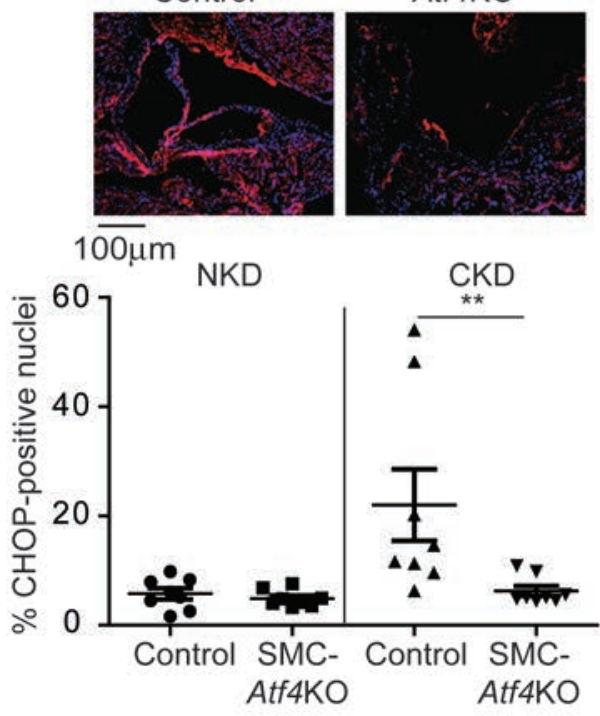

B

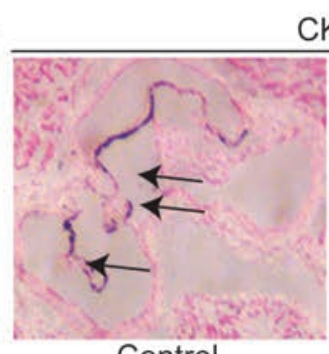

Control

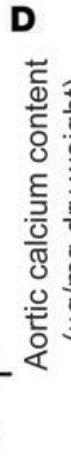

范

$\mathbf{F}$
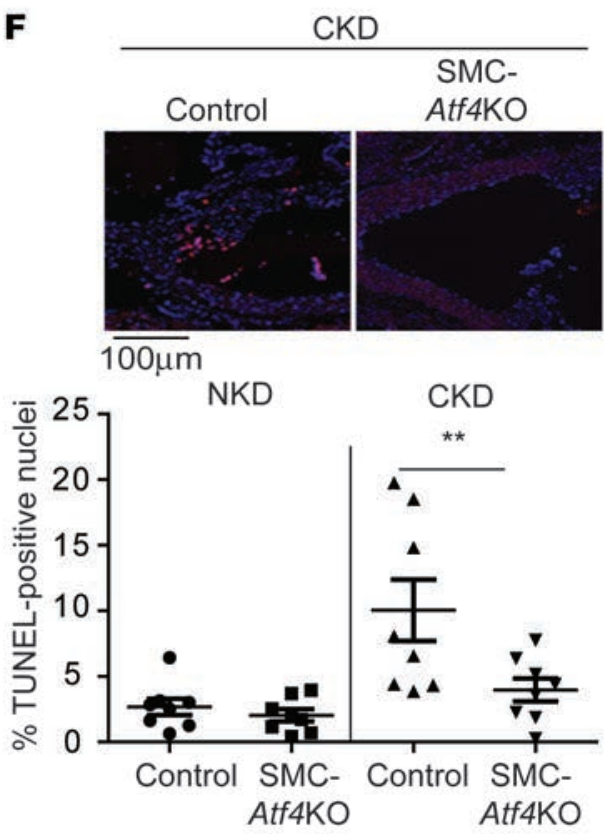

Figure 2. SMC-specific ATF4 deficiency attenuates medial calcification. (A) Immunoblot analysis of ATF4 in the medial layer of aortas of control and SMC-Atf4 KO mice on DBA/2] background. Five-week-old male mice were injected with either vehicle or $1 \mathrm{mg}$ tamoxifen for 5 consecutive days. Eight-weekold control and SMC-Atf4 KO mice $(n=8)$ were subjected to either sham operation for normal kidney condition (NKD) or $5 / 6$ $\mathrm{nx}$ for chronic kidney disease condition (CKD). The mice were sacrificed 12 weeks after the surgeries. (B) Representative photographs (original magnification, $\times 10$ ) of the lesions in aortic sinuses stained with von Kossa. Arrows denote calcified lesions. (C) Quantitative analysis of calcified lesions in the aortic sinus. More NKD data are shown in Supplemental Figure 2F.

(D) Aortic calcium content. (E) Immunofluorescence analysis of CHOP (red) in the aortic media of control and SMC-Atf4 KO mice. (F) Immunofluorescence-based TUNEL analysis of aortic media from control and SMC-Atf4 KO mice. Representative micrographs show less TUNEL-positive signal (pink) in nuclei (blue) of lesions from SMC-Atf4 KO mice under CKD than from control mice. One-way ANOVA with a Student-Newman post-hoc test was used for comparison between control and SMC-Atf4 KO mice. ${ }^{*} P<0.05,{ }^{* *} P<0.01$, ${ }^{* * *} P<0.001$. Scale bar: $100 \mu \mathrm{m}$.

and SMC-specific Atf4 KO; ApoE $E^{-/-}$mice under both NKD and CKD. As expected, CKD increased serum cholesterol, phosphorus, and creatinine in both control and SMC-specific Atf4 KO mice compared with NKD (Supplemental Figure 3).

SMC-specific ATF4 overexpression induces medial and atherosclerotic calcification. We next investigated whether CKD-mediated vascular calcification can be mimicked by SMC-specific ATF4 overexpression. The random insertion of genetic elements such as a transgene into the mouse genome may lead to the deregulation or misregulation of the expression of essential genes. The transgene may also be subject to a variegation or position effect. This ultimately leads to biased phenotype observations. To circumvent the random insertion of the floxed ATF4 transgene, a conditional human ATF4 transgene was inserted into a permissive locus, Rosa26, by the recombinase-mediated cassette exchange procedure that we recently developed (Figure 4A). Conditional $A T F 4$ transgenic (TG, Rosa26-ATF4 ${ }^{\text {oxtg/+ }}$ ) mice were bred with SMMHC-CreER ${ }^{(T 2)}$ mice to generate SMC-ATF4

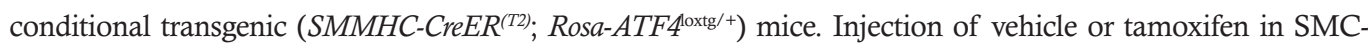
ATF4 conditional transgenic mice caused ATF4 overexpression (Figure 4B). Under NKD, control mice, as 
A

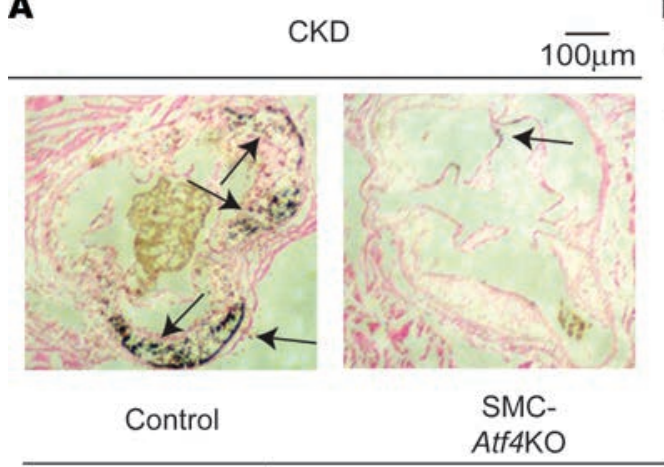

$A p o E^{-/}$background

\section{C}

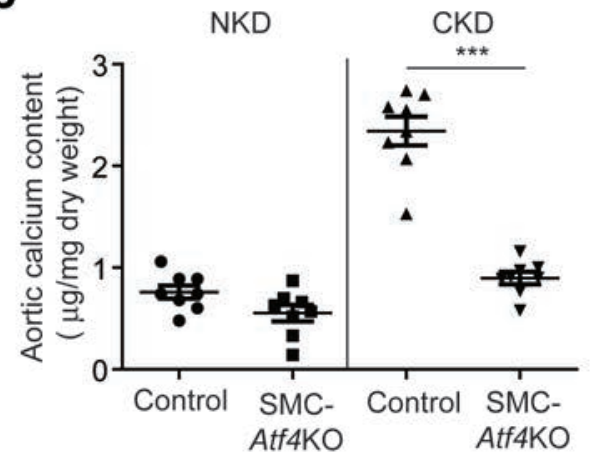

ApoE $E^{-/}$background
B

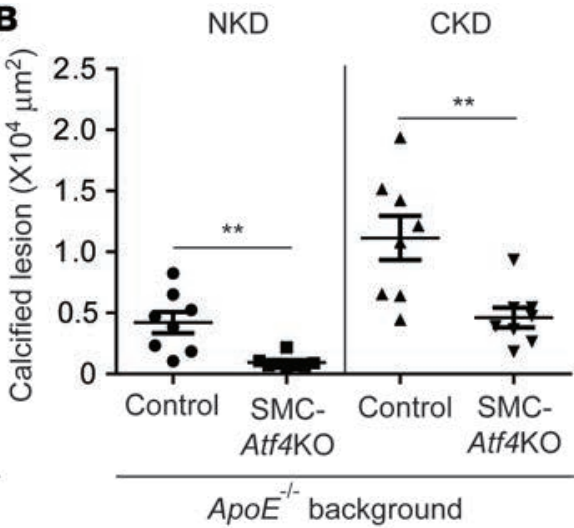

D

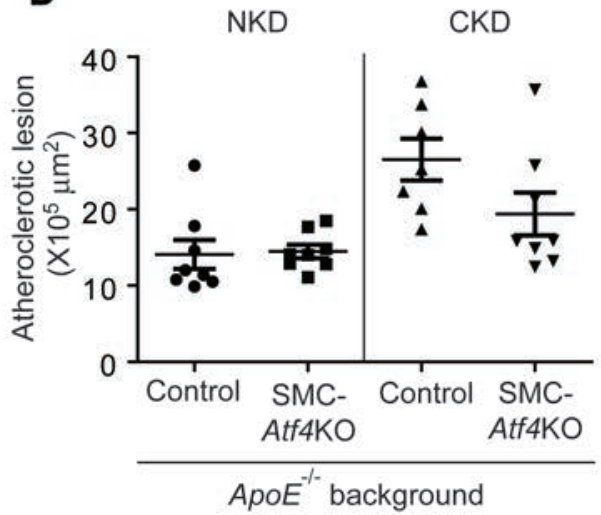

Figure 3. SMC-specific ATF4 deficiency attenuates atherosclerotic calcification but not atherosclerosis. (A) Representative photographs (original magnification, $\times 10$ ) of the lesions in aortic sinuses stained with von Kossa. Eight-week-old control and SMC-Atf4 KO mice $(n=8)$ on $C 57 \mathrm{BI} / 6 \mathrm{ApoE}^{-/-}$background (control ApoE $E^{-/-}$and SMC-Atf4KO; ApoE $E^{-/-}$mice) were subjected to either sham operation for normal kidney condition (NKD) or 5/6 nx for chronic kidney condition (CKD). The mice were sacrificed 12 weeks after the surgeries. Arrows denote calcified lesions. (B) Quantitative analysis of calcified lesions in the aortic sinus. (C) Aortic calcium content in control $A p o E^{-/-}$and SMC-Atf4KO; ApoE $E^{-/-}$mice under NKD and CKD. (D) Quantitative analysis of atherosclerotic lesions in the aortic sinus. Aortic sinuses were stained with Oil Red O. One-way ANOVA with a Student-Newman post-hoc test was used for statistical analysis. ${ }^{* *} P<0.01$, ${ }^{* *} P<0.001$. Scale bar: $100 \mu \mathrm{m}$.

expected, did not show mineral deposits in the aortic sinuses, whereas SMC-ATF4 TG mice displayed severe medial calcification (Figure 4, C and D). In addition, aortic calcium content was increased by 2.5 -fold in SMCATF4 TG mice compared with control mice (Figure 4E). SMC-ATF4 overexpression increased the number of apoptotic cells in the aorta (Figure 4, F and G). Serum parameters, including cholesterol, triglyceride, phosphorus, and calcium, were not altered by SMC-ATF4 overexpression (Supplemental Figure 4, A-D).

To further investigate whether ATF4 overexpression induces atherosclerotic calcification in addition to medial calcification, SMC-ATF4 TG mice were bred with $A p o E^{-/-}$mice to generate $S M M H C$-CreER ${ }^{(T 2)}$; Rosa$A T F 4^{\text {loxtg/++}}$; Apo $E^{-/-}$mice. SMMHC-CreER $R^{(T 2)} ;$ Rosa-ATF4 $4^{\text {loxtg/+}} ; A p o E^{-/-}$mice were intraperitoneally injected with vehicle or tamoxifen to generate control $A p o E^{-/-}$mice and SMC-specific ATF4 TG; ApoE ${ }^{-/-}$mice. SMC-ATF4 overexpression did not affect serum cholesterol, triglyceride, phosphorus, or calcium (Supplemental Figure 5, A-D) or atherosclerotic lesions (Supplemental Figure 5E). Histological analysis showed that SMC-specific ATF4 TG mice displayed severe vascular calcification compared with control ApoE $E^{-1-}$ mice (Supplemental Figure 5, F and G). In addition, SMC-specific ATF4 overexpression increased aortic calcium content by 1.9 -fold (Supplemental Figure $5 \mathrm{H}$ ).

SMC-ATF4 regulates the type III sodium-dependent phosphate cotransporters PiT1 and PiT2. Type III sodiumdependent phosphate transporters are considered major contributors to vascular calcification $(24,35-37)$. Our previous in vitro studies suggest that ATF4 may regulate vascular calcification through induction of phosphate transporter expression $(8,9)$. To examine this hypothesis, we first analyzed the expression of the type III sodium-dependent phosphate transporters SLC20A1 (PiT1) and SLC20A2 (PiT2) in the aortic medial layer of SMC-Atf4 KO and SMC-ATF4 TG mice. Both real-time quantitative PCR and immunoblot analysis revealed that ATF4 strongly regulates levels of both PiT1 and PiT2 expression in the medial layer of aortas. SMC-specific ATF4 deficiency significantly reduced levels of PiT1 and PiT2 mRNA and protein under both NKD and CKD conditions (Figure 5, A and B), whereas SMC-specific ATF4 overexpression induced mRNA and protein levels of both PiT isoforms (Figure 5, C and D). Consistently, VSMCs isolated from SMC-Atf4 KO mice had 71\% lower phosphate uptake than VSMCs from control mice (Figure $5 \mathrm{E}$ ). In addition, phosphate uptake of VSMCs increased by 2.7-fold in SMC-ATF4 TG mice (Figure 5F). 
A

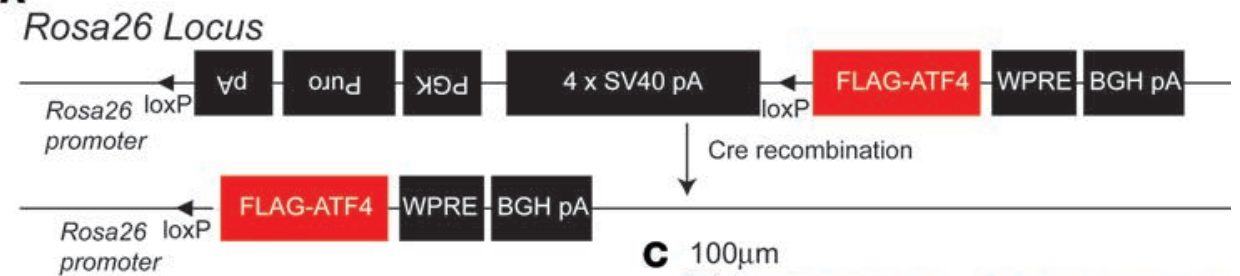

Figure 4. SMC-specific ATF4 overexpression induces medial calcification. (A) Scheme of targeting construct design for ATF4 transgenic (TC) in the Rosa26 locus. Triangles indicate the loxP sites. ATF4 conditional TC mice were backcrossed 10 times with DBA/2) mice. ATF4 conditional TC mice were crossed with SMMHC-Cre $e^{E R(T 2)}$ TC mice to generate SMC-ATF4 TC mice.

B

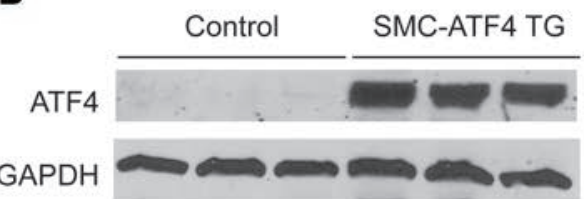

D

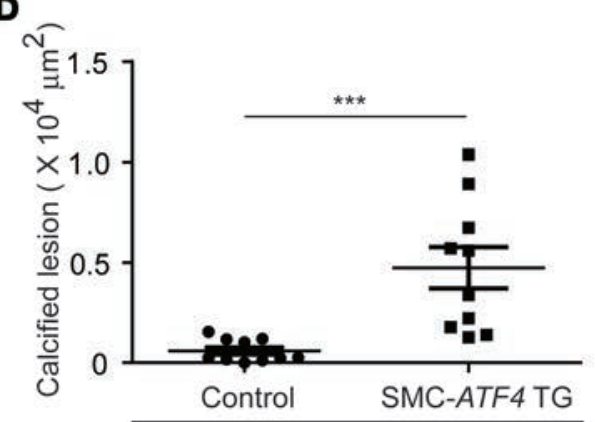

$\mathrm{DBA} / 2 \mathrm{~J}$ background

F

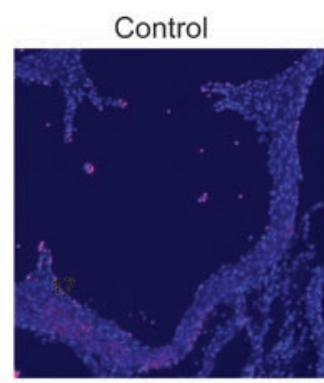

$\overline{100 \mu \mathrm{m}}$

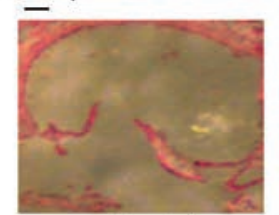

Control

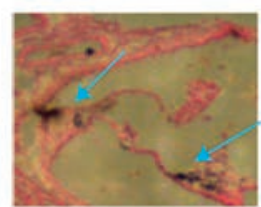

SMC-ATF4 TG

E

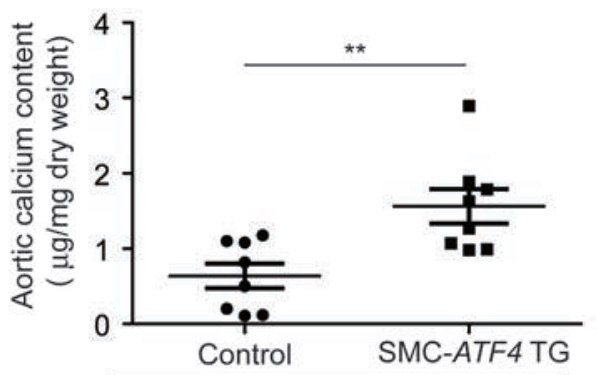

DBA/2J background

G

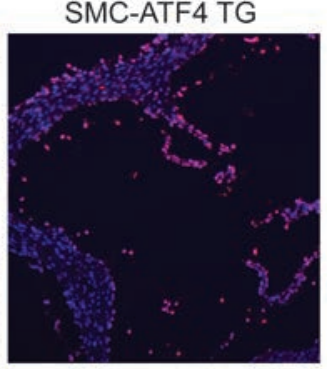

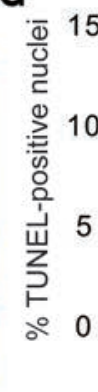

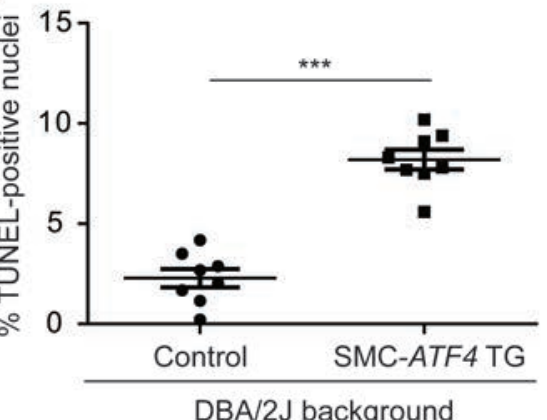

Five-week-old male mice under normal kidney conditions were injected with vehicle or $1 \mathrm{mg}$ tamoxifen for 5 consecutive days to generate control mice and SMC-ATF4 TC mice, respectively. The mice were sacrificed at 18 weeks of age. (B) Immunoblot analysis of ATF4 in the medial layer of aortas from control and SMC-ATF4 TG mice. (C) Representative photographs (original magnification, $\times 10$ ) of the lesions in aortic sinuses stained with von Kossa. (D) Quantitative analysis of calcified lesions in the aortic sinus. (E) Aortic calcium content in control and SMC-ATF4 TC mice. (F) Representative micrographs (original magnification, $\times 10$ ) show more TUNEL-positive signal (pink) in nuclei (blue) of aortic sinus lesions from SMC-ATF4 TC mice than from control mice. (G) Quantitative analysis of TUNELpositive nuclei conducted on aortic sinus lesions from SMC-ATF4 TC mice. Twotailed unpaired Student's $t$ test was used for statistical analysis. ${ }^{* *} P<0.01,{ }^{* *} P<$ 0.001 . Scale bar: $100 \mu \mathrm{m}$.

The ATF4-C/EBP $\beta$ complex regulates transcription of PiT1 and PiT2 genes. Since ATF4 is a pleiotropic transcription factor, we next proceeded to locate the ATF4 response element (ATF4RE) in the PiT1 gene locus. We generated a PiT1 reporter in which the luciferase reporters contained $1.4 \mathrm{~kb}$ and $964 \mathrm{bp}$ of the $5^{\prime}$ flanking region until exon 2 of the PiT1 gene, allowing the PiT1 gene to drive luciferase expression (Figure 6A). The PiT1 reporter construct was cotransfected with CMV-ATF4 into VSMCs. ATF4 expression led to an approximately 5.3-fold and 5.8-fold increase in luciferase activity with the $-617 /+825$ and $-139 /+825$ PiT1 constructs, respectively, but not with the $-617 /+86$ construct. These data suggest that the region between positions +86 and +825 contains regulatory sequences that mediate induction by ATF 4 . To further define the regulatory sequences responsible for ATF4 regulation, a homology search was performed using the consensus OSE1, cAMP response element (CRE), and ATF-C/EBP response sites, which are known to recruit ATF4. Computational analysis of the PiT1 promoter revealed a putative ATF4RE between positions +244 and +251 in intron 1 of the PiT1 gene, showing extensive homology to the ATF-C/EBP site previously identified in ATF4, VEGF, and CHOP genes $(38,39)$. To definitively demonstrate that the effect of ATF4 on the PiT1 gene is mediated through this putative ATF4RE, point mutations in the ATF4RE were generated in the +244 to +251 PiT1 construct and subjected to the same transfection assay. Mutations in the ATF4RE of intron 1 of PiT1 diminished the response to ATF4 overexpression (Figure 6A). Together, these results indicate that an ATF4RE is located in intron 1 of the PiT1 gene between positions +244 and +251 . 
A - Control - SMC-Atf4KO
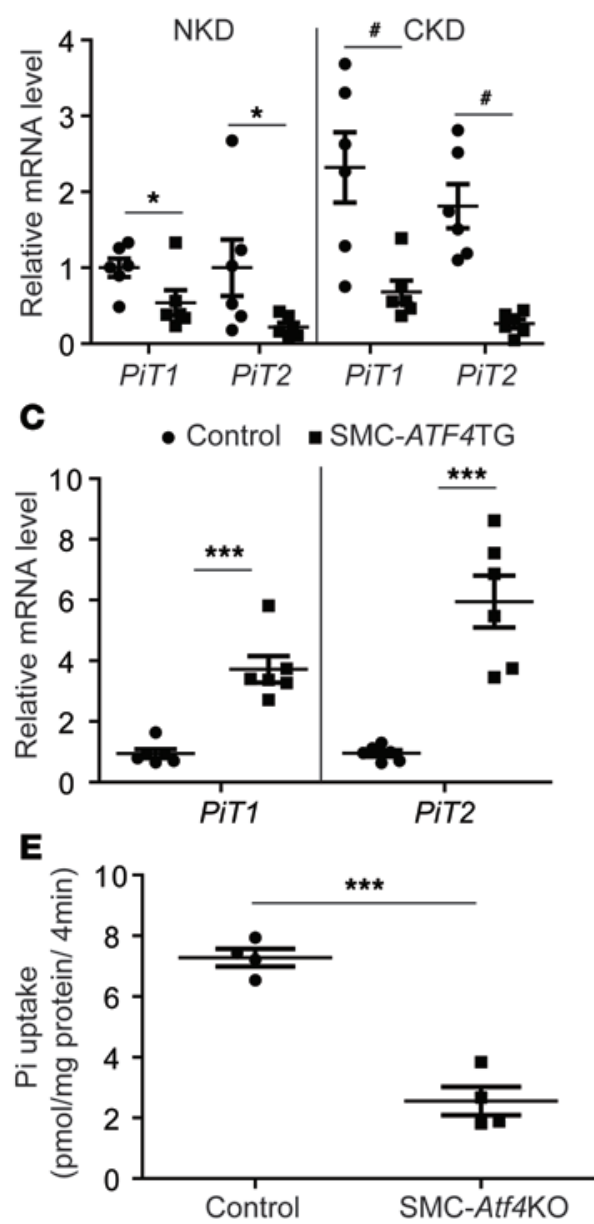

B

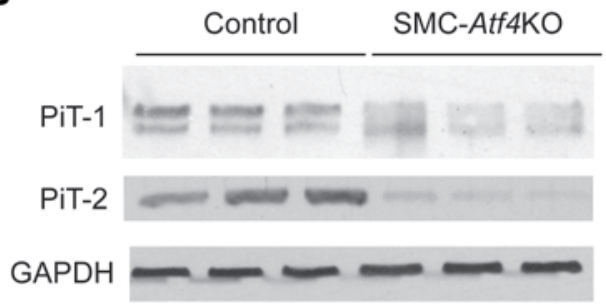

D

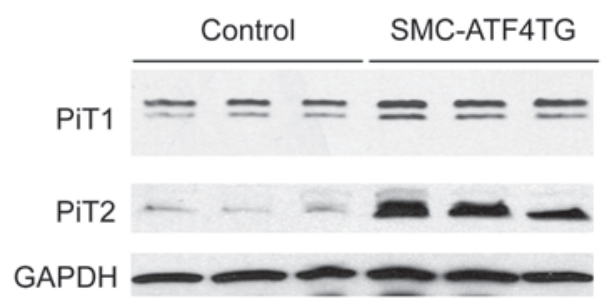

F

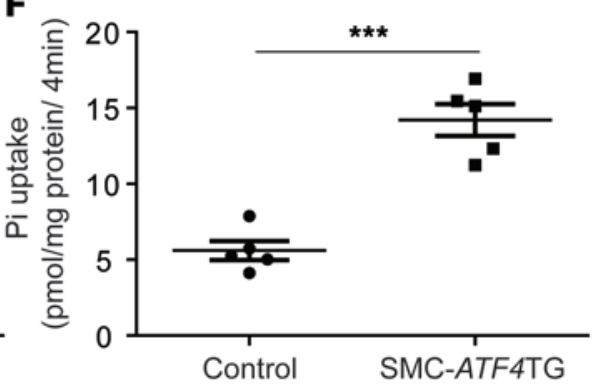

Figure 5. ATF4 regulates the aortic expression of PiT1 and PiT2. (A) Levels of PiT1 and PiT2 mRNA in the medial layer of aortas of control and SMC-Atf4 KO mice under normal kidney conditions (NKD) and chronic kidney disease conditions (CKD). (B) Immunoblot analysis of PiT1 and PiT2 in the medial layer of aortas of control and SMC-Atf4 KO mice under CKD. (C) Levels of PiT1 and PiT2 mRNA in the medial layer of aortas of control and SMC-ATF4 TC mice. (D) Immunoblot analysis of PiT1 and PiT2 in the medial layer of aortas of control and SMC-ATF4 TC mice. (E) Inorganic phosphate $(\mathrm{Pi})$ was analyzed in VSMCs isolated from the aortas of control and SMCAtf4 KO mice. (F) Pi uptake was analyzed in VSMCs isolated from the aortas of control and SMC-ATF4 TC mice. Two-tailed unpaired Student's $t$ test was used for statistical analysis. ${ }^{*} \mathrm{P}<0.05,{ }^{*} P<0.01,{ }^{* *} P<0.001$, $\# \mathrm{P}<0.05$

To determine whether ATF4 binds to the ATF4RE, gel mobility shift assays were performed using in vitro-translated ATF4 proteins and ${ }^{32} \mathrm{P}$-radiolabeled oligonucleotides corresponding to the PiT1 ATF4RE (Supplemental Figure 6A). Double-stranded radiolabeled oligonucleotides corresponding to the OSE1 of the osteocalcin promoter known to bind with the ATF4 homodimer were used as a control. As expected, the OSE of the osteocalcin promoter

made a shift with the ATF4 recombinant protein, which was blocked by excess WT OSE1 oligonucleotides but not the OSE1 mutant. However, the ATF4 protein did not bind to the ATF4RE of the PiT1 gene (Supplemental Figure 6A). These results led us to hypothesize that ATF4 requires another protein as a heterodimer partner to bind to the ATF4RE of the PiT1 gene.

To identify the heterodimer partner of ATF4 in VSMCs, we performed a yeast 2-hybrid screen using the ATF4 bZIP domain as bait and screened against a cDNA library. The library was made using RNA from VSMCs treated with stearic acid, which is known to induce ER stress and the subsequent induction of ATF4 and vascular calcification. As shown in Supplemental Table 1, we isolated 48 positive clones, which included 29 different genes, 4 of which are known to be transcription factors: C/EBP $\beta, \mathrm{C} / \mathrm{EBP} \gamma, \mathrm{NRF} 2$, and $\mathrm{Ybx} 1$ (Figure 6B and Supplemental Table 1). We chose C/EBP $\beta$ as a candidate for an ATF4 heterodimer partner for the transcriptional regulation of the PiT1 gene because the yeast 2-hybrid screen suggested that $\mathrm{C} / \mathrm{EBP} \beta$ is most abundantly associated with ATF4 in VSMCs (Supplemental Table 1) and because, similarly to ATF4, C/EBP $\beta$ translation is activated through the phosphorylation of eIF2 $\alpha$ during ER stress $(40,41)$. We therefore investigated whether the ATF4-C/EBP $\beta$ complex activates transcription of the PiT1 gene. Luciferase assay analysis showed that, while both ATF4 and C/EBP $\beta$ independently increased promoter activity of the PiT1 construct by 3-fold, when both ATF4 and C/EBP $\beta$ were coexpressed, the promoter activity was synergistically increased by 10 -fold (Figure 6C).

To confirm that ATF4-C/EBP $\beta$ heterodimers bind to the ATF4RE of the PiT1 gene, we performed EMSA (Figure 6D). Neither ATF4 nor C/EBP $\beta$ recombinant proteins bound alone to the ATF4RE of the PiT1 oligonucleotides, whereas, when both proteins were present, there was a shift in the band of radiolabeled oligonucleotides (Figure 6D), indicating binding of the ATF4-C/EBP $\beta$ heterodimer to the PiT1 WT ATF4RE. The addition of a 50-fold molar excess of unlabeled WT PiT1 ATF4RE abolished the shift, whereas unlabeled mutant PiT1 ATF4RE oligonucleotides did not (Figure 6D). In addition, C/EBP $\beta$ and 


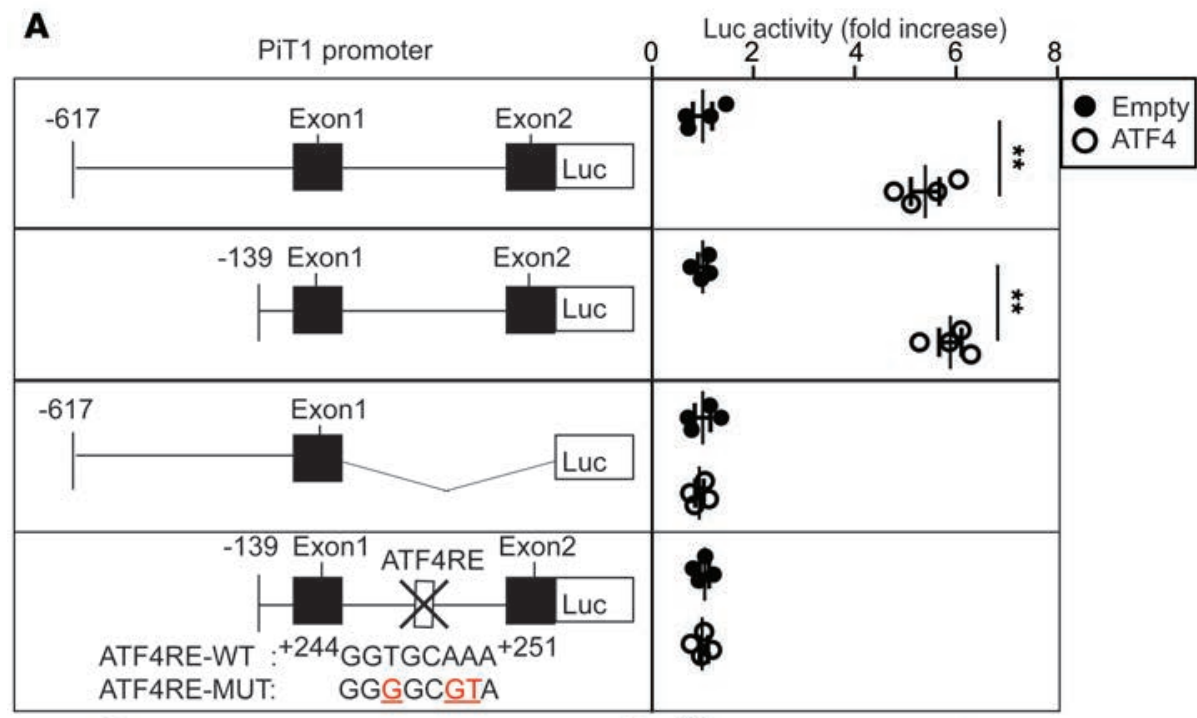

B

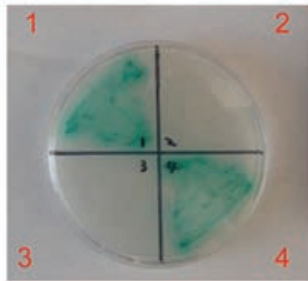

D

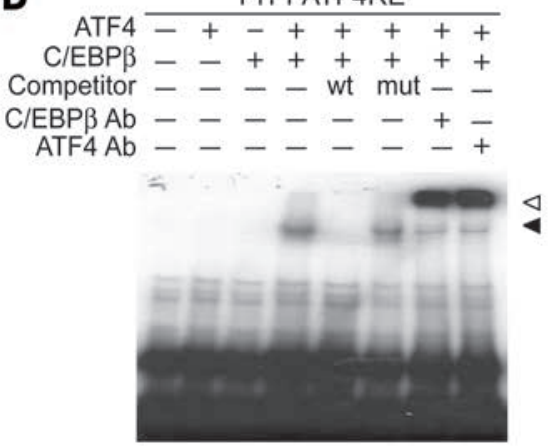

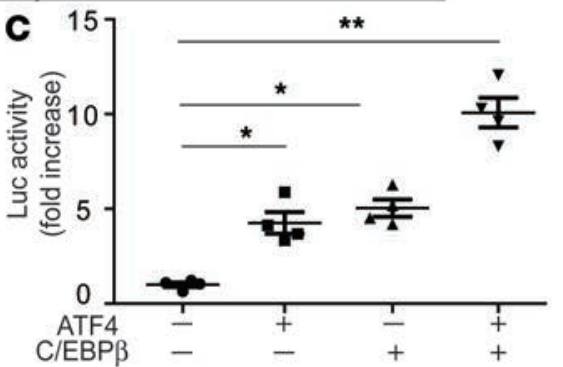

E

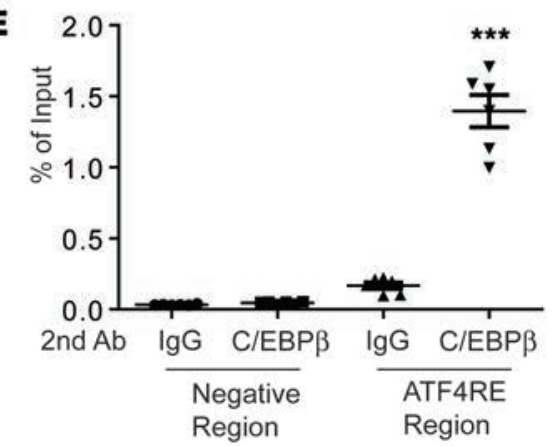

Figure 6. The transcriptional regulation of PiT1 by the ATF4-C/EBP $\beta$ complex. (A) Deletion and mutational analysis of the PiT1 gene using a luciferase (Luc) reporter gene assay. The schematic illustrations represent the serially deleted PiT/Luc reporter constructs. Results are expressed as the relative Luc/ $\beta$-galactosidase units of induction ( $n$-fold) over the control value for each construct. (B) Yeast 2-hybrid screen. Y2HGold yeasts expressing ATF4 and/or C/EBP $\beta$ were grown onto an SD agar plate placing 4 amino acids (Leu, Trp, Ade, and His) in the presence of X- $\alpha$-Gal and Aureobasidin A. Quadrant 1 of the plate contained a bait vector, pGBKT7-Atf4, and a prey vector, pGADT7-C/ebp $\beta$; quadrant 2 contained a bait vector, pGBKT7-Atf4, and a prey vector, pGADT7-Empty; quadrant 3 contained a bait vector, pGBKT7-C/ebp $\beta$, and a prey vector, pGADT7-Empty; and quadrant 4 contained a bait vector, pGBKT7-C/ebp $\beta$, and a prey vector, pGADT7-Atf4. (C) The ATF4-C/EBP $\beta$ complex induces PiT1 transcription in mammalian cells. NIH $3 T 3$ cells were cotransfected with pGL3 Luc plasmid containing the $-617 /+825$ PiT1 gene, ATF4-pcDNA3, and/or C/EBP $\beta$-pcDNA3 expression plasmid. (D) The ATF4-C/EBP $\beta$ complex but not ATF4 or C/EBP $\beta$ alone specifically binds to the PiT1 ATF4RE. EMSA was performed using ${ }^{32} \mathrm{P}$-radiolabeled double-stranded oligonucleotides corresponding to the ATF4RE of the PiT1 gene. Competitive assays were performed using a 50-fold molar excess of unlabeled oligonucleotides corresponding to the WT ATF4RE or the mutant ATF4 of the PiT1 gene as indicated. Supershift assay was performed using C/EBP $\beta$ and ATF4 antibody. Black arrow: binding ATF4-C/EBPbeta complex; white arrow: supershift. (E) ATF4 and C/EBP $\beta$ belong to the same protein complex, which binds to the PiT1 ATF4RE. Double ChIP assays were performed using VSMCs incubated for 6 hours with $500 \mu \mathrm{M}$ stearic acid that were first immunoprecipitated with ATF4 antibody, eluted, and then subjected to a second immunoprecipitation with C/EBP $\beta$ antibody or normal rabbit lgG antibody. The enrichment of ATF4-C/EBP $\beta$ protein was analyzed by real-time quantitative PCR using primer sets specific for the PiT1 ATF4RE or for the $5^{\prime}$ distal promoter region of PiT1 as a negative control (data not shown). Data were plotted as the percentage of antibody binding versus the amount of PCR product obtained using a standardized aliquot of input chromatin (\% of input). Two-tailed unpaired Student's $t$ test was used for statistical analysis. ${ }^{*} P<0.05,{ }^{* *} P<0.01,{ }^{* * *} P<0.001$. 


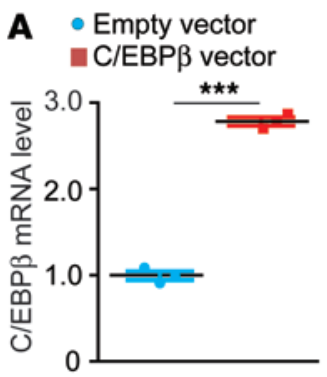

B
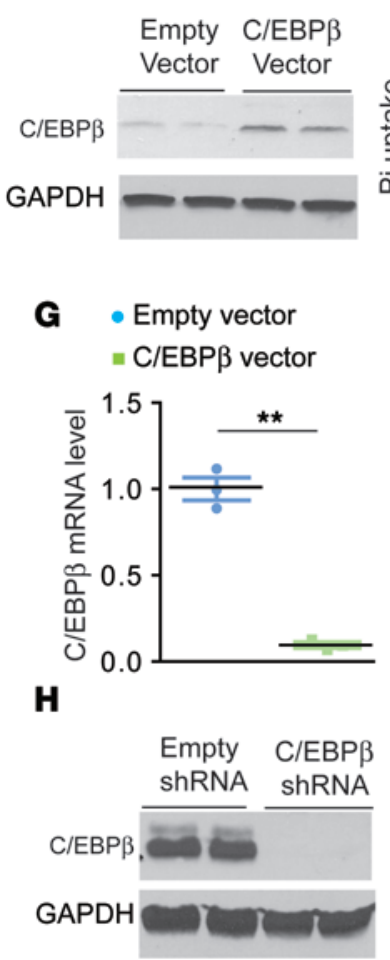
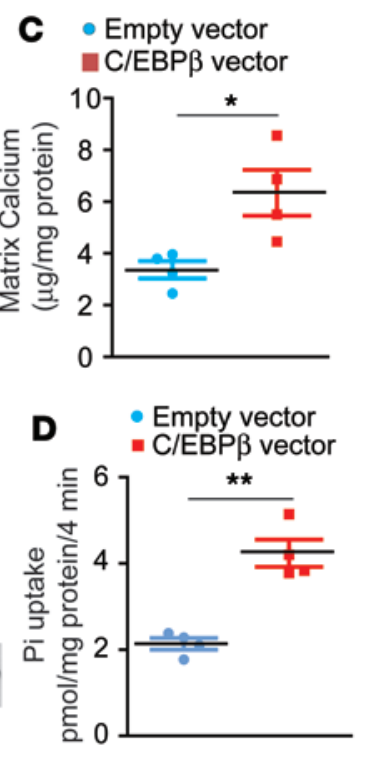

I Empty vector = $\mathrm{C} / \mathrm{EBP} \beta$ vector

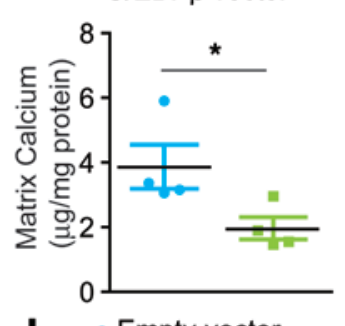

J

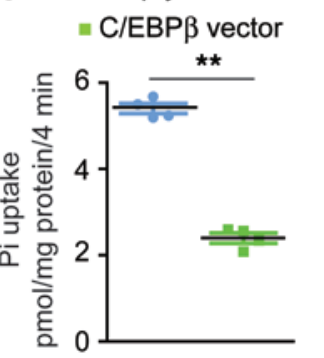

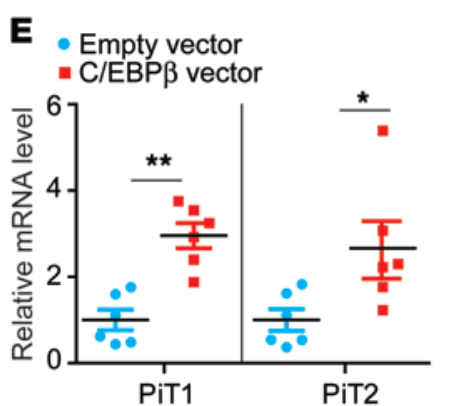
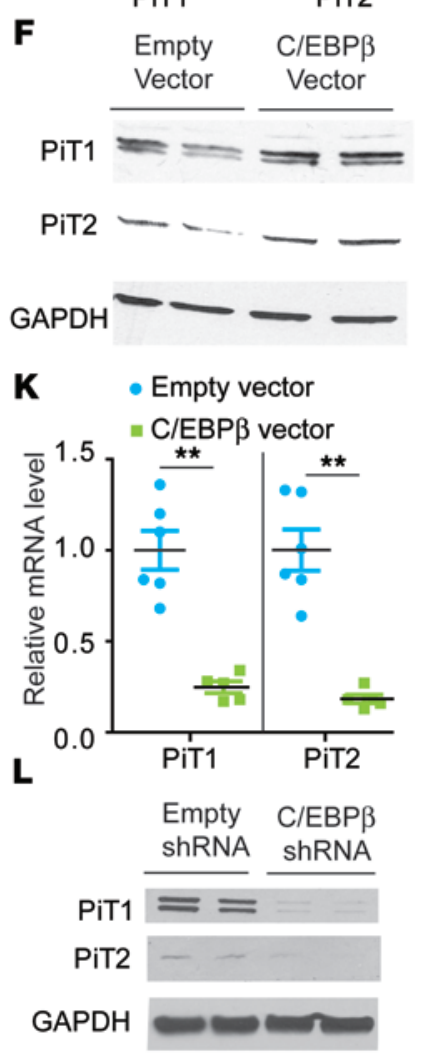

Figure 7. C/EBP $\beta$ regulates mineralization and phosphate uptake of VSMCs. Levels of C/EBP $\beta$ (A) mRNA and (B) protein in VSMCs overexpressing C/EBP $\beta$. VSMCs were infected with lentiviruses containing human $\mathrm{C} / \mathrm{EBP} \beta$. A single colony was isolated by puromycin selection. (C) Mineralization of VSMCs. VSMCs were incubated with $2.0 \mathrm{mM}$ phosphate for 7 days. (D) Inorganic phosphate (Pi) uptake in VSMCs overexpressing C/EBP $\beta$. Pi uptake was analyzed with ${ }^{32} \mathrm{P}-\mathrm{KH}_{2} \mathrm{PO}_{4}$. Levels of PiT1 and PiT2 (E) mRNA and $(\mathbf{F})$ protein in VSMCs overexpressing C/EBP $\beta$. Levels of C/EBP $\beta(\mathbf{C})$ mRNA and $(\mathbf{H})$ protein in C/ EBP $\beta$ knockdown VSMCs. The cells were infected with lentiviruses containing C/EBP $\beta$ shRNA. A single colony was isolated in the presence of puromycin. (I) Mineralization of VSMCs. VSMCs were incubated with $2.0 \mathrm{mM}$ phosphate for 7 days. (J) Pi uptake in C/EBP $\beta$ knockdown VSMCs. $\mathrm{Pi}$ uptake was analyzed with ${ }^{32} \mathrm{P}-\mathrm{KH}_{2} \mathrm{PO}_{4}$. Levels of PiT1 and PiT2 (K) mRNA and (L) protein in C/ EBP $\beta$ knockdown VSMCs. ${ }^{*} P<0.05$, ${ }^{* *} P<0.01$, ${ }^{* * *} P<0.001$. Two-tailed unpaired Student's $t$ test was used for statistical analysis

ATF4 antibodies produced a supershift of the band (Figure 6D), supporting that the complex included C/EBP $\beta$.

To further confirm whether ATF4 and $\mathrm{C} / \mathrm{EBP} \beta$ bind to the intronic ATF4RE of the PiT1 gene, a double ChIP analysis was performed in VSMCs that were treated overnight with $200 \mu \mathrm{M}$ stearate. The isolated chromatin was first immunoprecipitated with ATF4 antibody and then subjected to a second immunoprecipitation with $\mathrm{C} / \mathrm{EBP} \beta$ antibody or rabbit IgG. The double ChIP analysis confirmed ATF4-C/EBP $\beta$ binding to the region (Figure 6E). These results demonstrate the specific binding of the ATF4-C/EBP $\beta$

complex to the PiT1 ATF4RE. In addition, we also found that the ATF4-C/EBP $\beta$ complex activates the PiT2 promoter and that the ATF4RE is located in the PiT2 promoter between positions -127 and -111 (Supplemental Figure 6B).

$C / E B P \beta$ regulates mineralization and PiT1 and PiT2 expression in VSMCS. To further confirm the contribution of $\mathrm{C} / \mathrm{EBP} \beta$ in the regulation of vascular calcification and phosphate cotransporters, we generated stable VSMC lines overexpressing C/EBP $\beta$ (Figure 7, A and B). As expected, the overexpression of human $\mathrm{C} / \mathrm{EBP} \beta$ induced mineralization of VSMCs, along with significant increases in inorganic phosphate uptake and mRNA and protein levels of PiT1 and PiT2 (Figure 7, C-F). shRNA-mediated inhibition of C/EBP $\beta$, on the other hand, reduced mineralization, phosphate uptake, and PiT1 and PiT2 expression (Figure 7, G-L).

In addition, we recently reported that the saturated fatty acid stearic acid strongly induces vascular calcification through ER stress $(9,10)$. We then determined whether saturated fatty acids induce the expression of PiT1 and C/EBP $\beta$. Stearic acid treatment increased levels of PiT1 mRNA and C/EBP $\beta$ protein (Supplemental Figure 7, A and B) and induced vascular calcification (Supplemental Figure 7C) in control VSMCs. We next determined whether PiT1 contributes to stearate-induced vascular calcification. PiT1 shRNA treatment reduced PiT1 mRNA expression by 74\% (Supplemental Figure 7A). PiT1 knockdown significantly attenuated stearate-induced vascular calcification (Supplemental Figure 7C). 


\section{Discussion}

ATF4 is known to be a critical transcription factor that regulates skeletal osteogenesis and bone formation $(19,20,42,43)$. We and other investigators previously reported that ER stress induces expression of aortic ATF4 in a number of in vitro and in vivo models of vascular calcification (1-3, 5, 7-10). In particular, CKD strongly activates the aortic ER stress response, resulting in a significant induction of aortic ATF4 (8). In this study, we demonstrate that ATF4 expression in VSMCs plays a causative role in the pathogenesis of vascular calcification using a series of mouse models. As an initial model, we used global ATF4-haploinsufficient mice, which showed significantly smaller aortic medial calcified lesions under both CKD and NKD conditions. We also used an SMC-specific ATF4-deficient model, in which both medial and atherosclerotic calcifications under NKD and CKD conditions were attenuated. Finally, we generated a mouse model that overexpresses ATF4 only in SMCs, in which severe medial and atherosclerotic calcification developed even under NKD. These findings strongly suggest that the ectopic induction of ATF4 in VSMCs through ER stress is a pivotal event in the development of vascular calcification and osteogenesis.

We also provided mechanistic insights into ATF4-mediated vascular calcification. Our previous in vitro studies showed that shRNA-mediated Atf4 knockdown strikingly reduced mRNA levels of PiT1 and PiT2 in cultured VSMCs $(9,10)$. PiT1 and PiT2 are known to contribute to vascular calcification. Consistent with our previous in vitro studies, SMC-specific ATF4 deficiency in mice significantly reduced mRNA and protein levels of PiT1 and PiT2 in the medial layer of aortas, whereas SMC-specific ATF4 overexpression induced their levels. Using the promoter deletion assay, we identified ATF4REs in the PiT1 and PiT2 genes. The ATF4REs in PiT1 and PiT2 are similar to the ATF-C/EBP site previously identified in a number of genes involved in ER stress, including asparagine synthetase, VEGF, and CHOP genes $(38,39)$, locations where ATF4 binds. EMSA analysis, however, indicated that ATF4 protein alone does not bind to the ATF4REs of the PiT1 and PiT2 genes, although the OSE1 site in the osteocalcin promoter recruits an ATF4 homodimer, as previously reported $(19,44)$. These observations suggest the involvement of another protein in the regulation of PiT1 and PiT2 genes. Using yeast 2-hybrid screening, EMSA, and double ChIP analysis, we indicated that $\mathrm{C} / \mathrm{EBP} \beta$ most abundantly interacts with ATF4 and that the binding is increased though the ER stress response in VSMCs. Since the ATF4-C/EBP $\beta$ complex is known to promote osteoblast differentiation in bone formation, deletion of either the C/EBP $\beta$ or ATF4 gene from mice resulted in delayed bone formation, with concurrent suppression of chondrocyte maturation and osteoblast differentiation. $\mathrm{CKD}$ increases ATF4 and C/EBP $\beta$ protein levels through ER stress, suggesting that ectopic overproduction of the ATF4-C/EBP $\beta$ complex in VSMCs rather than ATF4 alone leads to vascular calcification through the induction of phosphate uptake. We also demonstrated that $\mathrm{C} / \mathrm{EBP} \beta$ regulates mineralization of VSMCs by altering PiT1 and PiT2 expression, similar to our previous observations on ATF4. Further studies will be required to confirm the in vivo contribution of $\mathrm{C} / \mathrm{EBP} \beta$ and the ATF4-C/EBP $\beta$ complex in the pathogenesis of vascular calcification by knocking down and overexpressing C/EBP $\beta$ in VSMCs. In addition to $\mathrm{C} / \mathrm{EBP} \beta$, at least 27 other proteins, including $\mathrm{C} / \mathrm{EBP} \gamma, \mathrm{NRF} 2$, and $\mathrm{YBX} 1$, interact with ATF4 in VSMCs. Further studies will be required to determine whether these proteins are involved in the regulation of vascular calcification.

CHOP is a major target of ATF4 (45), and it is a transcription factor that promotes apoptosis contributing to vascular calcification $(7,8)$. We previously reported that global $\mathrm{CHOP}$ deficiency attenuated CKD-dependent vascular apoptosis and atherosclerotic calcification in $A p o E^{-1-}$ mice (7). In this study, SMC-ATF4 deficiency reduced aortic CHOP expression and CKD-dependent apoptosis accompanied by a marked attenuation of vascular calcification, whereas SMC-ATF4 overexpression induced CHOP expression and apoptosis. A recent ChIP-seq study showed that the concomitant induction of ATF4 and CHOP makes a complex that mediates ER stress-mediated apoptosis $(38,46)$. CHOP also strongly interacts with $\mathrm{C} / \operatorname{EBP} \beta(47,48)$. Although our yeast 2-hybrid screen did not find an interaction between ATF4 and CHOP in VSMCs, it is still possible that ATF4, CHOP, and C/EBP $\beta$ cooperatively mediate CKD-dependent aortic apoptosis and vascular calcification. CHOP regulates the expression of PiT1 but not PiT2 by interacting with $\mathrm{C} / \mathrm{EBP} \beta$, which is mediated through the same DNA element of PiT1 (+243 to +251) that is regulated by the ATF4-C/EBP $\beta$ complex (data not shown). In addition, CHOP deficiency reduces aortic PiT1 mRNA and protein expression in vivo (7). These results suggest that ATF4 mediates vascular calcification through the induction of phosphate uptake in VSMCs through CHOP-dependent and -independent mechanisms.

In conclusion, using several mouse models, we have demonstrated a pivotal role of ATF4 in the pathogenesis of vascular calcification in vivo. In addition, our studies revealed that ATF4 regulation of 
phosphate uptake in VSMCs requires a heterodimer partner, C/EBP $\beta$. Therefore, targeting the ATF4-C/ $\mathrm{EBP} \beta$ complex and/or its regulating signals in VSMCs may represent a novel therapeutic strategy for attenuating vascular calcification.

\section{Methods}

Animals. Atf4 conditional KO ( $\left.{ }^{10 x / 10 x}\right)$ mice and $S M M H C$-Cre ${ }^{E R(T 2)}$ mice were generated as previously described $(10,49,50)$. SMMHC-Cre/GFP global Atf4 ${ }^{+/-}$and DBA/2J mice were obtained from The Jackson Laboratory $(51,52)$. For SMC-specific ATF4 TG mice, we introduced the transgene at the Rosa26 locus by the recombinase-mediated cassette exchange method that we recently developed (53) to circumvent the inherent problem of random insertion via traditional pronuclear injection. In brief, we transfected $5 \times 10^{6}$ R26FNF3-1F1 ES cells with $15 \mu \mathrm{g}$ of pFLSLF3-FLAG human ATF4 and $15 \mu \mathrm{g}$ of pCAG-Flpe (Addgene plasmid 13787). From the 96 clones tested, we found 7 clones with the expected genotype that were also G418 sensitive, indicating successful exchange of the original neo cassette for the transgene cassette at the Rosa26 locus. Karyotypically normal ES clones were microinjected into C57BL/6 blastocysts to produce chimeric founders at the University of Colorado Transgenic Animal Core Facility. The generated mice were named Rosa26-ATF4 conditional TG (Rosa26-ATF4 $4^{\text {oxtg/+}}$ ) mice. All of the mouse stains were backcrossed more than 10 times with DBA/2J and C57B1/6J mice. The genetic backgrounds were checked at the BioResources Core Facility of Barbara Davis Center at the University of Colorado Denver. The Atf $4^{\text {ox/lox }}$ and Rosa26-ATF $4^{\text {oxtg/+ }}$ mice were intercrossed with $S M M H C$-Cre ${ }^{E R(T 2)}$ mice to obtain $S M M H C-C r e^{E R(T 2)} ; A t f 4^{\text {tox/lox }}$ mice. Since the SMMHC-Cre $e^{E R(T 2)}$ transgene was inserted on the Y chromosome, only males were used in this study. Five-week-old males were intraperitoneally injected with either $1 \mathrm{mg}$ tamoxifen in vegetable oil or vehicle for 5 consecutive days to produce SMC-specific ATF4 KO/ TG mice and control mice, respectively. After the injections, the mice were maintained on a special diet (TD10364; Harlan Teklad) for 10 weeks. For VSMC isolation, Atf4 $4^{\text {lox/lox }}$ and Rosa26-ATF4 $4^{\text {loxtg/+ }}$ mice were intercrossed with constitutively active SMMHC-Cre/GFP mice. CKD was induced using 5/6 nx as previously reported, whereas sham operation was used as an $\operatorname{NKD}$ condition $(7,8,54)$.

Cell cultures. Murine aortic VSMCs (passages 6-10) were isolated from the aortas of control, SMCAtf4 KO, and SMC-ATF4 TG mice as described previously $(55,56)$. Cells were maintained in DMEM containing 10\% FBS. Mouse VSMCs (MOVAS-1, ATCC) were infected with recombinant lentiviruses containing $C / e b p \beta$ shRNA (clone TRCN0000007442, Open Biosystems), PiT1 shRNA (clone V2LMM_33774, Open Biosystems), and human $C / E B P \beta$ cDNA (Thermo Fisher). Colonies were selected by treatment with $5 \mu \mathrm{g} / \mathrm{ml}$ puromycin or blasticidin, respectively, for 7 days.

Histological analysis. Calcified lesions in the aortic sinus were analyzed as previously described (7, 10, 54). To distinguish between calcified lesions and pigments on the aortic valve leaflets, histological images were captured before and after von Kossa staining. Apoptotic cells and CHOP in the medial layer of aortic sinuses were detected using an In Situ Cell Death Detection Kit (Roche), a CD68 monoclonal antibody (FA-11, AbD Serotec), an $\alpha$-smooth muscle actin antibody (1A4, Sigma-Aldrich), an ATF4 monoclonal antibody (D4B8, Cell Signaling Technology), and a CHOP monoclonal antibody (L63F7, Cell Signaling Technology) as previously described $(7,8,57)$. At least 5 sections from each sample were analyzed.

Immunoblot analysis. Cell and tissue lysates were prepared using RIPA buffer (Cell Signaling). The samples were separated by SDS-PAGE, transferred to a nitrocellulose membrane, and immunoblotted with the following antibodies: ATF4 (D4B8) from Cell Signaling and PiT1 (H-130), PiT2 (B-4), C/EBP $\beta$ (C-19), and GAPDH (V-18) from Santa Cruz Biotechnology. Samples were visualized using horseradish peroxidase coupled to appropriate secondary antibodies, with enhancement by an ECL detection kit (Thermo Fisher Scientific).

Calcium content in cultured cells and aortas. Calcium deposition in cultured cells and aortas was quantified as previously described $(7-10,54)$.

Inorganic phosphate uptake measurement. Sodium-dependent uptake of phosphate was measured in VSMCs grown to confluency on 12-well plastic dishes as previously described $(8,9)$.

$R N A$ analysis. Real-time quantitative PCR assays were performed using an Applied Biosystems StepOne qPCR instrument. Quantitative expression values were calculated from an absolute standard curve method using the plasmid template (Open Biosystems) containing each target gene cDNA. Primer sequences that are fully validated were obtained from the Primer Bank (Harvard University, https://pga.mgh.harvard. edu/primerbank/) and published previously (10). 
Yeast 2-hybrid screen. Matchmaker library construction and screen kits were purchased from Clontech. A VSMC library was generated and the screen was performed as described in the manufacturer's protocols, using total RNA from VSMCs treated with $200 \mu \mathrm{M}$ stearic acid for 16 hours. Atf4 cDNA was subcloned into the pGBKT7 bait vector. Procedures for culture media, plates for yeast growth, and selection were followed as described in the manufacturer's protocols. Positive clones were sequenced and identified using the BLAST search on the UCSC Genome Bioinformatics (http://genome.ucsc.edu/).

Transfections and luciferase assays. NIH $3 \mathrm{~T} 3$ cells (ATCC) were grown at $37^{\circ} \mathrm{C}$ in an atmosphere of $5 \%$ $\mathrm{CO}_{2}$ in DMEM containing $25 \mathrm{mM}$ glucose, $100 \mathrm{U} / \mathrm{ml}$ penicillin, and $100 \mu \mathrm{g} / \mathrm{ml}$ streptomycin and supplemented with $10 \%$ fetal bovine serum. Transient transfections of NIH 3T3 cells were performed in triplicate in 24-well plates. Cells were transfected with $200 \mathrm{ng}$ of firefly luciferase reporter plasmids (pGL3, Promega), $50 \mathrm{ng}$ of expression plasmids, and $100 \mathrm{ng}$ of $\mathrm{pCMV}-L a c Z$ vector using Turbofect transfection reagent (Fisher). Six hours after transfection, cells were incubated in DMEM containing $10 \%$ fetal bovine serum. Luciferase activities were measured using a luciferase assay system (Promega). Firefly luciferase activity was divided by $\beta$-galactosidase activity to obtain a normalized value, the relative luciferase unit. Expression plasmids for human ATF4 (plasmid 26114) and $C / E B P \beta$ (plasmid 12558) were purchased from Addgene.

In vitro transcription/translation and EMSA. Human ATF4 and C/EBP $\beta$ proteins were synthesized in vitro from $\mathrm{pGEM}-A T F 4$ and $\mathrm{pGEM}-C / E B P \beta$ using the TNT quick-coupled transcription/translation system (Promega) as previously described (58). Double-stranded oligonucleotides corresponding to the ATF4 RE of the PiT1 gene (PiT1 WT ATF4RE, 5'-GCGGCACCGGTGGTGCAAAGGTCCTTTCT-3') and the OSE of the osteocalcin promoter (5'-CTCCCCTGCTCCTCCTGCTTACATCAGAGAGCACA-3') were ${ }^{32} \mathrm{P}$ radiolabeled with polynucleotide kinase. Protein-DNA-binding assays were performed by incubating the protein and radiolabeled probes at room temperature for 20 minutes, followed by 15 minutes on ice. Salmon sperm DNA was added into the binding assays to reduce nonspecific binding of labeled oligonucleotides. A 50-fold molar excess of unlabeled double-stranded mutant PiT1 ATF4RE gene (PiT1 Mut ATF4RE, 5'-GCGGCACCGGTGGGGCGTAGGTCCTTTCT-3') or unlabeled double-stranded mutant OSE1 of the osteocalcin promoter (Mut OSE1, 5'-CTCCCCTGCTCTTGGAGCATGCATCAGAGAGCACA-3') was used for the competition experiments. The underlines denote nucleotide mutations. The DNA-protein complexes were resolved on a $5 \%$ polyacrylamide gel in $1 \mathrm{M}$ Tris-borate-EDTA buffer. The gels were dried and autoradiographed at $-80^{\circ} \mathrm{C}$.

Double ChIP analysis. ChIP was performed using an ATF4 antibody that was incubated for 30 minutes at $37^{\circ} \mathrm{C}$ in elution buffer $(0.01 \%$ SDS, $1.1 \%$ Triton X-100, $1.2 \mathrm{mM}$ EDTA, $16.7 \mathrm{mM}$ Tris-HCl, pH 8.0, 167 $\mathrm{mM} \mathrm{NaCl}, 10 \mathrm{mM}$ DTT). The supernatant was diluted 100 -fold with ChIP dilution buffer ( $25 \mathrm{mM}$ Tris- $\mathrm{HCl}$, pH 8.0, 2 mM EDTA, $150 \mathrm{mM} \mathrm{NaCl}, 1 \%$ Triton X-100) and then subjected to a second immunoprecipitation using either $\mathrm{C} / \mathrm{EBP} \beta$ antibody or normal rabbit IgG antibody. Purified immunoprecipitated DNA was analyzed by real-time quantitative PCR. The results are expressed as the percentage of antibody binding versus the amount of PCR product obtained using a standardized aliquot of input chromatin ( $\%$ of input). The primers for the PiT1 gene are as follows: sense, 5'-TGCTTCACGAGTGGGTAGAG-3', and antisense, 5'-GCCCACTCCAGAGAAGAAAG-3'. The following primers were used as a negative control: sense, 5'-GGAGAAAGCACTTGAGAGGAATA-3', and antisense, 5'-GCAGTCAGAGGAATGAGTGTAG-3'.

Biochemical analysis. Serum cholesterol, triglyceride, phosphorus, and calcium were analyzed using colorimetric enzyme assay kits (Pointe Scientific, cholesterol C7510; triglyceride T7532; phosphorus P7516; calcium C7503). Serum creatinine was analyzed with LC-MS/MS as previously described $(7,10)$.

Statistics. Data were collected from more than 2 independent experiments and are reported as mean \pm SEM. Statistical analysis was performed using a 2-tailed Student's $t$ test for 2-group comparisons and a 1-way ANOVA with a Student-Newman post-hoc test or a 2-way ANOVA for multigroup comparison. Significance was accepted at $P<0.05$.

Study approval. All animal protocols and experimental procedures were approved by the Institutional Animal Care and Use Committees at the University of Colorado Denver.

\section{Author contributions}

M. Masuda, SMA, and M. Miyazaki conceptualized the study. M. Masuda, SMA, YT, and M. Miyazaki developed the methodology. M. Masuda, SMA, and M. Miyazaki formally analyzed the data. M. Masuda, SMA, and M. Miyazaki curated data. M. Masuda, SMA, ALK, YS, KO, and KW investigated the literature. M. Masuda, KW, and M. Miyazaki wrote the original draft. ALK, YT, WSC, and 
M. Miyazaki reviewed and edited the manuscript. M. Masuda, SMA, and M. Miyazaki generated the figures. M. Masuda and M. Miyazaki acquired funding. SMR, WSC, KW, XZ, YT, and CMA provided resources. M. Miyazaki supervised the work.

\section{Acknowledgments}

The authors' work was supported by grants from the NIH (R01DK096030, R01HL117062, and R01 HL132318 to M. Miyazaki). M. Masuda received a fellowship from the American Heart Association (13POST13820008).

Address correspondence to: Makoto Miyazaki, 12700 East 17th Avenue C281 RC-2 Room 7450, Aurora, Colorado 80045, USA. Phone: 303.724.4828; E-mail: makoto.miyazaki@ucdenver.edu.

1. Cai Z, et al. Endoplasmic reticulum stress participates in aortic valve calcification in hypercholesterolemic animals. Arterioscler Thromb Vasc Biol. 2013;33(10):2345-2354.

2. Duan XH, et al. Activating transcription factor 4 is involved in endoplasmic reticulum stress-mediated apoptosis contributing to vascular calcification. Apoptosis. 2013;18(9):1132-1144.

3. Liberman M, Johnson RC, Handy DE, Loscalzo J, Leopold JA. Bone morphogenetic protein-2 activates NADPH oxidase to increase endoplasmic reticulum stress and human coronary artery smooth muscle cell calcification. Biochem Biophys Res Commun. 2011;413(3):436-441.

4. Mizobuchi M, Towler D, Slatopolsky E. Vascular calcification: the killer of patients with chronic kidney disease. $J$ Am Soc Nephrol. 2009;20(7):1453-1464.

5. Duan X, Zhou Y, Teng X, Tang C, Qi Y. Endoplasmic reticulum stress-mediated apoptosis is activated in vascular calcification. Biochem Biophys Res Commun. 2009;387(4):694-699.

6. Shao JS, et al. Vascular Bmp Msx2 Wnt signaling and oxidative stress in arterial calcification. Ann N Y Acad Sci. 2007;1117:40-50.

7. Miyazaki-Anzai S, et al. Endoplasmic reticulum stress effector CCAAT/enhancer-binding protein homologous protein (CHOP) regulates chronic kidney disease-induced vascular calcification. J Am Heart Assoc. 2014;3(3):e000949.

8. Masuda M, Miyazaki-Anzai S, Levi M, Ting TC, Miyazaki M. PERK-eIF2 $\alpha$-ATF4-CHOP signaling contributes to TNF $\alpha$ induced vascular calcification. J Am Heart Assoc. 2013;2(5):e000238.

9. Masuda M, Ting TC, Levi M, Saunders SJ, Miyazaki-Anzai S, Miyazaki M. Activating transcription factor 4 regulates stearateinduced vascular calcification. J Lipid Res. 2012;53(8):1543-1552.

10. Masuda M, et al. Saturated phosphatidic acids mediate saturated fatty acid-induced vascular calcification and lipotoxicity. $J$ Clin Invest. 2015;125(12):4544-4558.

11. Tabas I, Ron D. Integrating the mechanisms of apoptosis induced by endoplasmic reticulum stress. Nat Cell Biol. 2011;13(3):184-190.

12. Walter P, Ron D. The unfolded protein response: from stress pathway to homeostatic regulation. Science. 2011;334(6059):10811086 .

13. Harding HP, et al. An integrated stress response regulates amino acid metabolism and resistance to oxidative stress. $\mathrm{Mol}$ Cell. 2003;11(3):619-633.

14. Hai T, Hartman MG. The molecular biology and nomenclature of the activating transcription factor/cAMP responsive element binding family of transcription factors: activating transcription factor proteins and homeostasis. Gene. 2001;273(1):1-11.

15. Harding HP, et al. Regulated translation initiation controls stress-induced gene expression in mammalian cells. Mol Cell. 2000;6(5):1099-1108.

16. Sowa H, Karsenty G. ATF4 is a key molecule linking food intake and skeletal development. J Musculoskelet Neuronal Interact. 2007;7(4):326-327.

17. Xiao G, et al. Cooperative interactions between activating transcription factor 4 and Runx $2 /$ Cbfa1 stimulate osteoblast-specific osteocalcin gene expression. J Biol Chem. 2005;280(35):30689-30696.

18. Yang X, Karsenty G. ATF4, the osteoblast accumulation of which is determined post-translationally, can induce osteoblastspecific gene expression in non-osteoblastic cells. J Biol Chem. 2004;279(45):47109-47114.

19. Yang X, et al. ATF4 is a substrate of RSK2 and an essential regulator of osteoblast biology; implication for Coffin-Lowry Syndrome. Cell. 2004;117(3):387-398.

20. Saito A, et al. Endoplasmic reticulum stress response mediated by the PERK-eIF2(alpha)-ATF4 pathway is involved in osteoblast differentiation induced by BMP2. J Biol Chem. 2011;286(6):4809-4818.

21. Shanahan CM, Crouthamel MH, Kapustin A, Giachelli CM. Arterial calcification in chronic kidney disease: key roles for calcium and phosphate. Circ Res. 2011;109(6):697-711.

22. Cheng SL, et al. Targeted reduction of vascular Msx1 and Msx2 mitigates arteriosclerotic calcification and aortic stiffness in LDLR-deficient mice fed diabetogenic diets. Diabetes. 2014;63(12):4326-4337.

23. Sun Y, et al. Smooth muscle cell-specific runx2 deficiency inhibits vascular calcification. Circ Res. 2012;111(5):543-552.

24. Hu MC, et al. Klotho deficiency causes vascular calcification in chronic kidney disease. J Am Soc Nephrol. 2011;22(1):124-136.

25. Lai CF, Shao JS, Behrmann A, Krchma K, Cheng SL, Towler DA. TNFR1-activated reactive oxidative species signals up-regulate osteogenic Msx2 programs in aortic myofibroblasts. Endocrinology. 2012;153(8):3897-3910.

26. Demer LL, Tintut Y. Inflammatory, metabolic, and genetic mechanisms of vascular calcification. Arterioscler Thromb Vasc Biol. 2014;34(4):715-723.

27. Tintut Y, Patel J, Parhami F, Demer LL. Tumor necrosis factor-alpha promotes in vitro calcification of vascular cells via the 
cAMP pathway. Circulation. 2000;102(21):2636-2642.

28. Watson KE, Boström K, Ravindranath R, Lam T, Norton B, Demer LL. TGF-beta 1 and 25-hydroxycholesterol stimulate osteoblast-like vascular cells to calcify. J Clin Invest. 1994;93(5):2106-2113.

29. Giachelli CM, Speer MY, Li X, Rajachar RM, Yang H. Regulation of vascular calcification: roles of phosphate and osteopontin. Circ Res. 2005;96(7):717-722.

30. El-Abbadi MM, et al. Phosphate feeding induces arterial medial calcification in uremic mice: role of serum phosphorus, fibroblast growth factor-23, and osteopontin. Kidney Int. 2009;75(12):1297-1307.

31. New SE, Aikawa E. Cardiovascular calcification: an inflammatory disease. Circ J. 2011;75(6):1305-1313.

32. Proudfoot D, Skepper JN, Hegyi L, Bennett MR, Shanahan CM, Weissberg PL. Apoptosis regulates human vascular calcification in vitro: evidence for initiation of vascular calcification by apoptotic bodies. Circ Res. 2000;87(11):1055-1062.

33. Tintut Y, Patel J, Territo M, Saini T, Parhami F, Demer LL. Monocyte/macrophage regulation of vascular calcification in vitro. Circulation. 2002;105(5):650-655.

34. Aikawa E, et al. Osteogenesis associates with inflammation in early-stage atherosclerosis evaluated by molecular imaging in vivo. Circulation. 2007;116(24):2841-2850.

35. Voelk1 J, et al. Spironolactone ameliorates PIT1-dependent vascular osteoinduction in klotho-hypomorphic mice. J Clin Invest. 2013;123(2):812-822.

36. Crouthamel MH, et al. Sodium-dependent phosphate cotransporters and phosphate-induced calcification of vascular smooth muscle cells: redundant roles for PiT-1 and PiT-2. Arterioscler Thromb Vasc Biol. 2013;33(11):2625-2632.

37. Li X, Yang HY, Giachelli CM. Role of the sodium-dependent phosphate cotransporter, Pit-1, in vascular smooth muscle cell calcification. Circ Res. 2006;98(7):905-912.

38. Su N, Kilberg MS. C/EBP homology protein (CHOP) interacts with activating transcription factor 4 (ATF4) and negatively regulates the stress-dependent induction of the asparagine synthetase gene. J Biol Chem. 2008;283(50):35106-35117.

39. Oyadomari S, Mori M. Roles of CHOP/GADD153 in endoplasmic reticulum stress. Cell Death Differ. 2004;11(4):381-389.

40. Oyadomari S, Harding HP, Zhang Y, Oyadomari M, Ron D. Dephosphorylation of translation initiation factor 2alpha enhances glucose tolerance and attenuates hepatosteatosis in mice. Cell Metab. 2008;7(6):520-532.

41. Calkhoven CF, Müller C, Leutz A. Translational control of C/EBPalpha and C/EBPbeta isoform expression. Genes Dev. 2000;14(15):1920-1932.

42. Karsenty G. Transcriptional control of skeletogenesis. Annu Rev Genomics Hum Genet. 2008;9:183-196.

43. Wang X, et al. miR-214 targets ATF4 to inhibit bone formation. Nat Med. 2013;19(1):93-100.

44. Ducy P, Zhang R, Geoffroy V, Ridall AL, Karsenty G. Osf2/Cbfa1: a transcriptional activator of osteoblast differentiation. Cell. 1997;89(5):747-754.

45. Fawcett TW, Martindale JL, Guyton KZ, Hai T, Holbrook NJ. Complexes containing activating transcription factor (ATF)/ cAMP-responsive-element-binding protein (CREB) interact with the CCAAT/enhancer-binding protein (C/EBP)-ATF composite site to regulate Gadd153 expression during the stress response. Biochem J. 1999;339 (Pt 1):135-141.

46. Han J, et al. ER-stress-induced transcriptional regulation increases protein synthesis leading to cell death. Nat Cell Biol. 2013;15(5):481-490.

47. Shirakawa K, et al. CCAAT/enhancer-binding protein homologous protein (CHOP) regulates osteoblast differentiation. $M o l$ Cell Biol. 2006;26(16):6105-6116.

48. Ron D, Habener JF. CHOP, a novel developmentally regulated nuclear protein that dimerizes with transcription factors C/EBP and LAP and functions as a dominant-negative inhibitor of gene transcription. Genes Dev. 1992;6(3):439-453.

49. Ebert SM, et al. Stress-induced skeletal muscle Gadd45a expression reprograms myonuclei and causes muscle atrophy. $J$ Biol Chem. 2012;287(33):27290-27301.

50. Wirth A, et al. G12-G13-LARG-mediated signaling in vascular smooth muscle is required for salt-induced hypertension. Nat Med. 2008;14(1):64-68.

51. Masuoka HC, Townes TM. Targeted disruption of the activating transcription factor 4 gene results in severe fetal anemia in mice. Blood. 2002;99(3):736-745.

52. Xin HB, Deng KY, Rishniw M, Ji G, Kotlikoff MI. Smooth muscle expression of Cre recombinase and eGFP in transgenic mice. Physiol Genomics. 2002;10(3):211-215.

53. Williams K, Zhao X, Chick WS. A toolkit for transgensis at the ROSA26 locus by recombinase-mediated cassette exchange [version 1; referees: 1 approved with reservations, 2 not approved]. F1000Research. 2013;2:162. doi: 10.12688/f1000research.2-162.v1.

54. Miyazaki-Anzai S, Levi M, Kratzer A, Ting TC, Lewis LB, Miyazaki M. Farnesoid X receptor activation prevents the development of vascular calcification in ApoE-/- mice with chronic kidney disease. Circ Res. 2010;106(12):1807-1817.

55. Ting TC, et al. Increased lipogenesis and stearate accelerate vascular calcification in calcifying vascular cells. J Biol Chem. 2011;286(27):23938-23949.

56. Geng Y, et al. Role of cellular cholesterol metabolism in vascular cell calcification. J Biol Chem. 2011;286(38):33701-33706.

57. Thorp E, Li G, Seimon TA, Kuriakose G, Ron D, Tabas I. Reduced apoptosis and plaque necrosis in advanced atherosclerotic lesions of Apoe-/- and Ldlr-/- mice lacking CHOP. Cell Metab. 2009;9(5):474-481.

58. Chu K, Miyazaki M, Man WC, Ntambi JM. Stearoyl-coenzyme A desaturase 1 deficiency protects against hypertriglyceridemia and increases plasma high-density lipoprotein cholesterol induced by liver X receptor activation. Mol Cell Biol. 2006;26(18):6786-6798. 